\title{
Acquiring Accurate Body Measurements on a Smartphone from Supplied Colored Garments for Online Apparel Purchasing Platforms and E-Retailers
}

\author{
Sibei XIA, Andre WEST, Cynthia ISTOOK, Jiayin LI \\ The College of Textiles, North Carolina State University, Raleigh (NC) USA
}

DOI: $10.15221 / 18.126$ http://dx.doi.org/10.15221/18.126

\begin{abstract}
Return rates for e-retail fashion companies are significantly higher than in-store sales. Twenty to fifty percent of online clothing sales are returned. Apparel retailers are haunted by returns based on sizing issues, with $\$ 62.4$ billion in returns attributed to poor choices by the consumer in the USA. However, over the next ten years online sales are predicted to double, compounding the problem exponentially.
\end{abstract}

Garment sizing and knowing your correct size for a particular garment or brand while online shopping is part of the problem. It is the combinations of body measurements that determine sizing and sizing labels in clothing not usually one measurement. Most consumers don't know their body measurements when attempting to determine the size of a garment that they would like to purchase when shopping online and can have significant difficulty attempting to take their own measurements. This can lead to frustration and an incomplete sale or shopping cart abandonment. Many customers even resort buying a garment in two or more sizes and return the ones that do not fit, as they do not want to waste their time trying to determine which would be a perfect size. This adds to cost and waste affecting profitability. By the time these garments are returned to the vendor or manufacture they are out of season and usually not resalable at the original price because of the time lag and subsequent repackaging problems.

This research focuses on creating a fast-personal garment apparatus, system, and method for measuring body dimensions extracted from two-dimensional (2D) images captured by a consumer. Measurements of the individual are taken from captured pictures or photographs from their smart phones while wearing one or more coded dimensioning garments that have markings at specific locations that can be aligned with characteristic body features and key measurement areas. Computer vision is used to track these markings and extract key body dimensions. TensorFlow, a machine learning software application, is incorporated for object detection can be used to recognize colors and patterns on the garment allowing the garment to act as a measurement device for the body. The extracted dimensions could further used to predict additional body information such as; size growth and fit information, for example with fitness apps and workout appeal, or simply predicting children's wear and maternity wear needs as the body grows.

Keywords: 3D body scanning, e-commerce, body measurement

\section{Introduction}

Return rates for e-retail fashion companies are significantly higher than in-store sales. Twenty to fifty percent of online clothing sales are returned (Figure 1) [1,2,3]. Apparel retailers are haunted by returns based on sizing issues, with $\$ 62.4$ billion in returns attributed to poor choices by the consumer. However, over the next ten years online sales are predicted to double, compounding the problem exponentially [2].

Many issues have not been considered while developing size selection solutions. All consumers have body changes that occur over time that they may or may not be aware of, which can have a significant impact on the size of clothing needed. In addition, many customers are dissatisfied with the fit of the garments that they purchase, but "make do" because they can't find their exact fit. Some consumers want to know their exact size, while others just want something that fits. Consumers may need accurate personalized measurements for high-end merchandise, made to measure, tailored, bespoke customized, or special occasion garments (weddings). Therefore, there is an opportunity to develop a garment system that can be used by customers to measure themselves accurately, easily, and costeffectively and track this data. 
U.S. Apparel Market \$225 billion in 2012
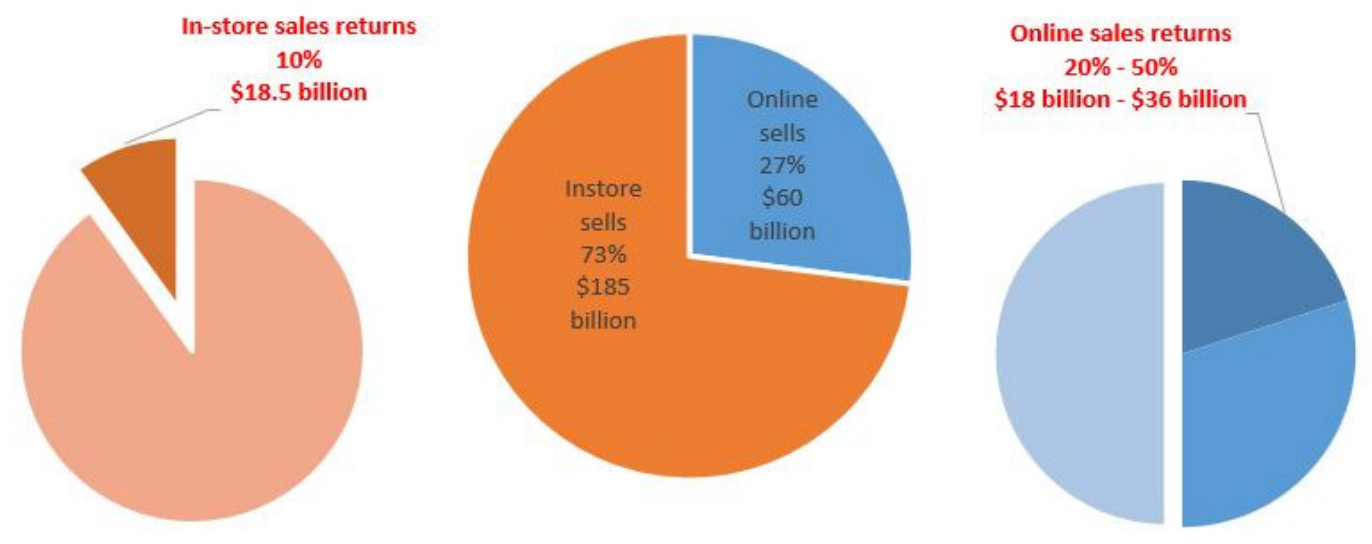

Figure 1. Return Rates of Clothing Industry [1, 2, 3].

There are many ways to collect body measurements. Traditionally, body measurements are collected manually through tape measures, anthropometers, and calipers $[4,5,6]$. However, this process is time-consuming and has low consistency $[7,8]$. A modern way of measuring bodies is through three-dimensional (3D) whole-body scanners. This method is faster and more accurate than the manual methods $[9,10,11]$. However, the cost of a 3D whole-body scanner is relatively high which makes it not applicable for daily use by apparel shoppers.

Although these machines are very accurate, they have not found their place in the retail environment and may actually be a hindrance to the satisfying shopping experience consumers usually want. There are also some concerns from the customers regarding the privacy of the data. Since the majority of the most accurate scanners aren't able to "see" through clothing, the less clothing worn by the consumers, the more accurate the result may be $[12,13]$. Many consumers are uncomfortable with the idea of images being captured of them in their underwear and do not want pictures of them circulating on the internet. There is also significant concern about children's images that could be used for nefarious purposes. With the increasing scanning speed, handheld scanners have grown in popularity and can potentially be used to measure a body but again the scanners need to be aware of or compensate for the clothing being worn. The garment being worn affects the measurement, to a significant degree.

Numerous consumer acts in each country are making it much harder to collect data that is not created voluntarily by consumers, themselves.[14] The technology that we are presenting here has the opportunity to provide consumers with the ability to measure their selves and not use these more obtrusive devices. They can use a personal smart device that not only extracts important measurements, but also has the ability to maintain the information over time.

Technology is the core to how millennials and the new I Generation (born since 1995) operate in the world. They communicate almost entirely online using their phones for everything [15]. Today retailers are also focused on the surge in mobile transactions. Because consumers want to use their cellphones all the time, the choice of using a cellphone as the body measurement device makes it convenient and easily accessible. However, since the RGB camera on a cellphone is no better at "seeing" through clothing than any other device in the marketplace, the reliability of the result relies heavily on the garment worn by the user. The development of a garment that can be easily photographed using a cell phone, or another device, which also might aid in the extraction of measurements could encourage consumers to use the technology without privacy concerns. The mobile app we have in development obtains measurements from 2D images that have been taken with a smart device (or regular digital camera). The software uses gender, height, weight, age, ethnicity, and the 2D measures to provide an output of significant girth and length dimensions to help consumers identify their "size" for clothing purchases. The output measures are accurate within the tolerances generally used for clothing production. 


\section{Measure through 2D images}

Two-dimensional (2D) images have become a popular solution for capturing body information because the capturing device has low cost, the images can provide acceptable results and image analyzing application programming interfaces (APIs) have become more powerful and accessible $[16,17,18,19]$. The 2D cameras can't capture as much information as the 3D whole body scanner when only a few images are taken. However, information collected through these 2D images can be used to derive unknown information using different methods, such as parameterized 3D templates and statistical prediction models $[20,21]$. However, $2 \mathrm{D}$ image body measuring techniques have not been fully commercialized, yet.

Location when the picture was taken, can be a limiting factor when trying to capture a body measurement from a 2D image. Most published research has found that there are many requirements for separating background colors, in order to be able to segment bodies from backgrounds $[22,23)$. Privacy is also still a concern [24]. To help solve these problems, apparatuses have been designed and tested [25]. These apparatuses can protect customer's privacy by covering the face and make it easier to identify body measurements. However, these devices are not consumer friendly.

With 2D images, the assistance of a garment apparatus, data mining and image processing techniques, it is possible to develop a body measurement system that can be used by customers to monitor their own measurements. Support statements from user interviews and background surveys previously conducted suggest that it would be valuable and feasible to develop a body measuring system that customers could use whenever and wherever they want to get more accurate body measurements than they would be likely to obtain using a measuring tape on their own.

\section{Research}

This research focused on creating a fast-personal apparatus, system, and method for measuring body dimensions extracted from two-dimensional (2D) images captured by a user. Measurements of the individual were taken from captured pictures or photographs while wearing one or more coded dimensioning garments designed by the team, with markings at specific locations that can be aligned with characteristic body features. Computer vision was used to track these markings and extract key body dimensions. The extracted dimensions were further used to predict additional body information. A large-scale three-dimensional anthropometric body database has been used to train the prediction models using machine-learning algorithms. A schematic of the system is illustrated in Figure 2 (a). A prototype of the apparatus is shown in Figure 2 (b).

The purpose of this design is to provide a possible solution for fast and easy body measurement calculation while making the garment both practical and aesthetically pleasing. Combined with certain algorithms, these garments can be used to establish body dimensions extracted from two-dimensional (2D) images captured by a smartphone or other digital device. These garments are marked at specific locations to align with characteristic body features and coded with different colors so the measurement system can recognize where to measure from the two-dimensional images. Camouflage patterns are applied in each color block to make the design less monotonous while ensuring the accuracy of measurements. Computer vision is used to track these markings and extract key body dimensions. The extracted dimensions are further used to predict additional body information.

The garments that have been developed can be kept, shared, returned or even worn as regular sports clothing. In e-retail, these garments can be shipped in the same box as a regular order. They can be used by a friend or family member and for all ages and populations with slight adjustments in size ranges. Once the garment pictures are taken, the retailer has access to the personal measurements of their customer, making targeted marketing easier.

Advantages of this system include: 1) accurate measurement results can be achieved within the general production tolerances of most apparel products; 2) data is obtained in an automatic format with better accuracy than manual body measurements taken by untrained consumers; 3 ) privacy concerns can be reduced by not using partially undressed pictures over the internet; 4) digital data is obtained in a format to facilitate size prediction in e-commerce transactions;5) potential reduction in returned merchandise due to sizing issues; and 6) no need for specialized facilities or expensive equipment, such as 3D scanners, to take body measurements. The garments are made with $83 \%$ Polyester $17 \%$ elastane four-way stretch fabric and the patterns are printed using digital printing technology. These garments are not only essential to the success of the software, they can also be used for active apparel. 


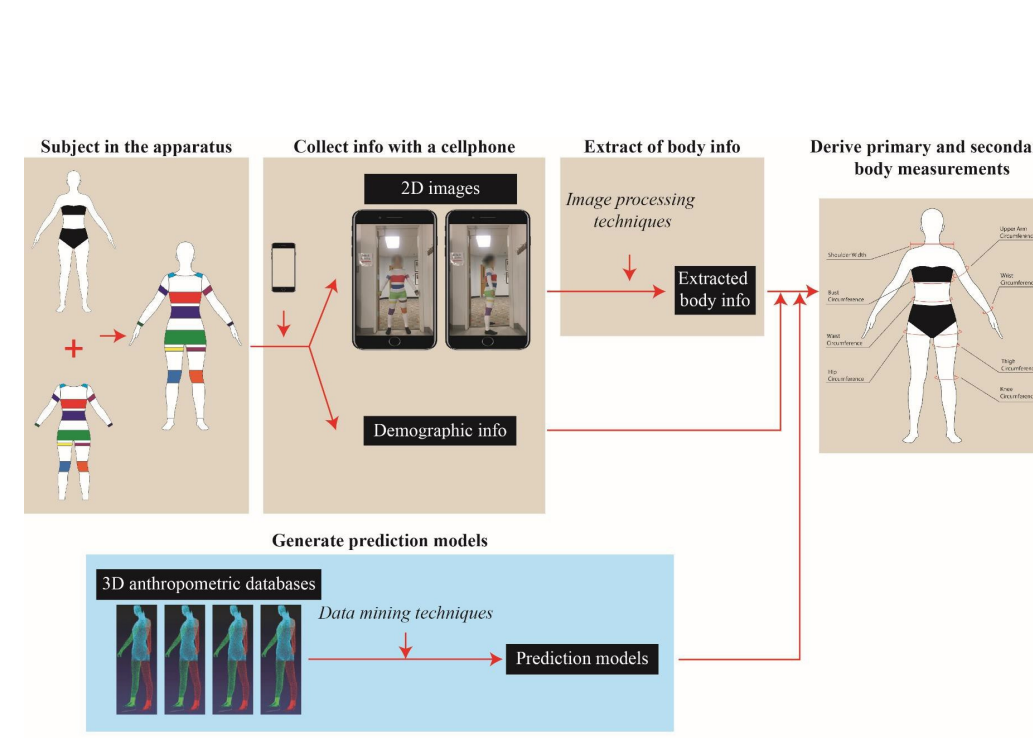

(a)

Figure 2. Schematic and prototype.

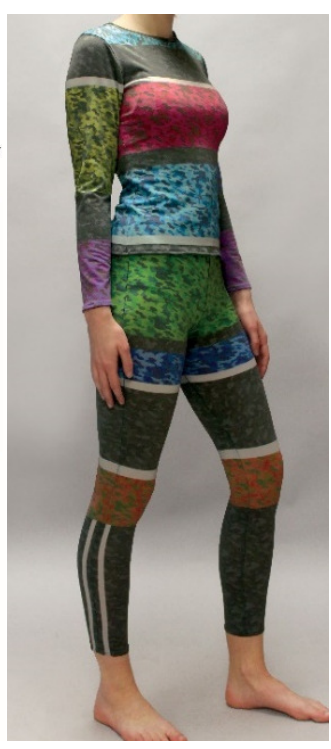

(b)

\section{Future developments}

The system that we developed is an efficient and cost-effective way to measure a body. Because most clothing shoppers have access to cellphones that can take pictures, it costs almost nothing for them to use it. In an optimal situation, most of the cost could be borne by the garment manufacturers but result in increased sales and substantially fewer returns due to fit issues. Most smart phones have internet access which offers further opportunities regarding using the body information. Customers can access their up-to-date body measurements at anytime and anywhere. The body measurements collected through the system could be used to select garment sizes, create online 3D avatars, customize clothing patterns, and track customer's fitness. The new system solves issues related to existing systems such as the high cost of 3D whole-body scanning technology, the measurement extraction dilemma of handheld scanners, and the low-quality results from manual measuring techniques. Overall, the developed body measuring system is innovative and important for the apparel industry, but also has the potential for the health industry.

\section{References}

[1] Pulga, A. (2015, August 11). How E-tailers Personalize Outfits to Customers' Taste. Retrieved February 27, 2018, from http://www.iqmetrix.com/blog/how-e-tailers-personalize-outfits-tocustomers-taste

[2] Cheng, A. (2015, June 8). Consumers return $\$ 642.6$ billion in goods each year. Retrieved February 27, 2018, from http://www.marketwatch.com/story/consumers-return-6426-billion-ingoods-each-year-2015-06-18

[3] Shah, S. (2015, October 30). Apparel industry: How many garments are sold globally online and offline per year? - Quora. Retrieved February 27, 2018, from https://www.quora.com/Apparelindustry-How-many-garments-are-sold-globally-online-and-offline-per-year

[4] Bye, E., Labat, K. L., \& Delong, M. R. (2006). Analysis of body measurement systems for apparel. Clothing and Textiles Research Journal, 24(2), 66-79

[5] Kidwell, C. B., \& Christman, M. C. (1974). Suiting everyone: The democratization of clothing in America (Vol. 5176). National Museum of History and Technology

[6] Roebuck, J. A. (1995). Anthropometric methods: designing to fit the human body. Human Factors and Ergonomics Society. 
[7] Bond, T. (2008). Computerised pattern making in garment production. In C. Fairhurst (Ed.), Advances in Apparel Production, Woodhead, Cambridge (pp. 140-153). Cambridge, England: Woodhead Publishing.

[8] Gill, S. (2015). A review of research and innovation in garment sizing, prototyping and fitting. Textile Progress, 47(1), 1-85.

[9] Daanen, H. A., \& Ter Haar, F. B. (2013). 3D whole body scanners revisited. Displays, 34(4), 270275.

[10] Gill, S. (2015). A review of research and innovation in garment sizing, prototyping and fitting. Textile Progress, 47(1), 1-85.

[11] Istook, C. L. (2008). Three-dimensional body scanning to improve fit. In C. Fairhurst (Ed.), Advances in apparel production (pp. 94-116). Cambridge, England: Woodhead Publishing

[12] Grogan, S., Siddique, M. A., Gill, S., Brownbridge, K., Storey, E., \& Armitage, C. J. (2017). 'I think a little bit of a kick is sometimes what you need': Women's accounts of whole-body scanning and likely impact on health-related behaviours. Psychology \& Health, 1-18

[13] Loker, S., Cowie, L., Ashdown, S., \& Lewis, V. D. (2004). Female consumers' reactions to body scanning. Clothing and Textiles Research Journal, 22(4), 151-160.

[14] Fleishman, G.(2018) California Passes Groundbreaking Consumer Data Privacy Law With Fines for Violations Retrieved September 3, 2018, from http://fortune.com/2018/06/28/californialaw-consumer-privacy-gdpr-fines-violations/

[15] Twenge, J.M. (2017). I-Gen: Why today's super-connected kids are growing up less rebellious, more tolerant, less happy--and completely unprepared for adulthood--and what that means for the rest of us. New York: Atria Press.

[16] Daanen, H. A., \& Ter Haar, F. B. (2013). 3D whole body scanners revisited. Displays, 34(4), 270275

[17] Lin, Y.-L., \& Wang, M.-J. J. (2011). Automated body feature extraction from 2D images.

[18] Expert Systems with Applications, 38(3), 2585-2591.

[19] Saito, S., Kouchi, M., Mochimaru, M., \& Aoki, Y. (2012). Simple system for 3D body shape estimation. In Consumer Electronics (GCCE), 2012 IEEE 1st Global Conference on (pp. 203205). IEEE. Retrieved from http://ieeexplore.ieee.org/abstract/document/6379580/

[20] Seo, H., Yeo, Y. I., \& Wohn, K. (2006). 3D body reconstruction from photos based on range scan. In International Conference on Technologies for E-Learning and Digital Entertainment (pp. 849-860). Springer. Retrieved from https://link.springer.com/chapter/10.1007/11736639_105

[21] Meunier, P., \& Yin, S. (2000). Performance of a 2D image-based anthropometric measurement and clothing sizing system. Applied Ergonomics, 31(5), 445-451.

[22] 19 Seo, H., Yeo, Y. I., \& Wohn, K. (2006). 3D body reconstruction from photos based on range scan. In International Conference on Technologies for E-Learning and Digital Entertainment (pp. 849-860). Springer. Retrieved from https://link.springer.com/chapter/10.1007/11736639_105

[23] Lin, Y.-L., \& Wang, M.-J. J. (2011). Automated body feature extraction from 2D images. Expert Systems with Applications, 38(3), 2585-2591.

[24] Seo, H., Yeo, Y. I., \& Wohn, K. (2006). 3D body reconstruction from photos based on range scan. In International Conference on Technologies for E-Learning and Digital Entertainment (pp. 849860). Springer. Retrieved from https://link.springer.com/chapter/10.1007/11736639_105

[25] Meunier, P., \& Yin, S. (2000). Performance of a 2D image-based anthropometric measurement and clothing sizing system. Applied Ergonomics, 31(5), 445-451. 